\author{
Guest Editors: Jose C. Nicolau, Claudia F Gravina and Roberto A. Franken
}

\title{
Transcatheter aortic valve replacement in elderly patients
}

\author{
Dimytri Siqueira $^{1}$, Alexandre Abizaid ${ }^{1}$, Magaly Arrais ${ }^{1}$, J. Eduardo Sousa ${ }^{1,2}$ \\ ${ }^{1}$ Institute Dante Pazzanese of Cardiology, Av. Dr Dante Pazzanese, 500 Ibirapuera, São Paulo, CEP 04012080, Brazil \\ ${ }^{2}$ Heart Hospital, Rua Desembargador Eliseu Guilherme, 147 Paraíso São Paulo, CEP 040004030, Brazil
}

\begin{abstract}
Aortic stenosis is the most common native valve disease, affecting up to $5 \%$ of the elderly population. Surgical aortic valve replacement reduces symptoms and improves survival, and is the definitive therapy in patients with symptomatic severe aortic stenosis. However, despite the good results of classic surgery, risk is markedly increased in elderly patients with co-morbidities. Transcatheter aortic valve replacement (TAVR) allows implantation of a prosthetic heart valve within the diseased native aortic valve without the need for open heart surgery and cardiopulmonary bypass, offering a new therapeutic option to elderly patients considered at high surgical risk or with contraindications to surgery. To date, several multicenter registries and a randomized trial have confirmed the safety and efficacy of TAVR in those patients. In this chapter, we review the background and clinical applications of TAVR in elderly patients.
\end{abstract}

J Geriatr Cardiol 2012; 9: 78-82. doi: 10.3724/SP.J.1263.2011.12291

Keywords: Aortic stenosis; Elderly patients; Transcatheter aortic valve replacement; Percutaneous valve therapies

\section{Introduction}

Valve disease prevalence is strongly associated with population aging. Currently, aortic stenosis is the most common native valve disease, affecting up to $5 \%$ of the elderly population. ${ }^{[1]}$ Surgical aortic valve replacement reduces symptoms and improves survival, and is the definitive therapy in patients with symptomatic severe aortic stenosis. ${ }^{[2]}$ However, despite the good results of classic surgery, risk is markedly increased in elderly patients with co-morbidities. Moreover, several registries have demonstrated that up to $30 \%$ of cases are considered too high risk for conventional open heart surgery and remain untreated, thus experiencing a poor prognosis. ${ }^{[3]}$

First performed in 2002, ${ }^{[4]}$ transcatheter aortic valve replacement (TAVR) allows implantation of a prosthetic heart valve within the diseased native aortic valve without the need for open heart surgery and cardiopulmonary bypass, offering a new therapeutic option to elderly patients considered at high surgical risk or with contraindications to surgery.

Correspondence to: Alexandre Abizaid, MD, PhD, Institute Dante Pazzanese of Cardiology, Av. Dr Dante Pazzanese, 500 Ibirapuera, São Paulo, CEP 04012080, Brazil. E-mail: aabizaid@uol.com.br

Telephone: +551150856315

Fax: $+55-1150856315$

Received: December 29, 2011

Revised: April 23, 2012

Accepted: April 30, 2012

Published online: June 9, 2012

\section{Currently available transcatheter heart valves}

Globally, approximately 40,000 patients were treated to date with this novel technique. Two percutaneous heart valves are currently available and marketed in Europe and around the world. The Edwards-SAPIEN valve system (Edwards Lifesciences Inc., Irvine, CA, USA) is a tri-leaflet bovine pericardial valve mounted on a balloon-expandable stent (Figure $1 \mathrm{~A}$ and $2 \mathrm{~B}$ ). The SAPIEN XT ${ }^{\circledR}$ valve, the third generation of the Edwards valve, is available in $20 \mathrm{~mm}$, $23 \mathrm{~mm}, 26 \mathrm{~mm}$, and $29 \mathrm{~mm}$ sizes. The CoreValve system (Medtronic, Minneapolis, MN) is a tri-leaflet porcine pericardial valve mounted in a self-expandable nitinol stent (Figure $1 \mathrm{~B}$ and 2A). The valve is available in $26 \mathrm{~mm}, 29 \mathrm{~mm}$, and $31 \mathrm{~mm}$ sizes. Both devices are usually implanted by a transfemoral retrograde approach; alternative access includes trans-apical and trans-aortic approaches for the SAPIEN ${ }^{\mathrm{R}}$ valve and transaortic and trans-subclavian approaches for the CoreValve ${ }^{\circledR}$.

Numerous different percutaneous aortic valves are in clinical evaluation. The development of new valves is sufficiently justified and underscores the need to reduce delivery catheter diameter, to ensure accurate annular positioning, to reduce the occurrence of para-valvular leaks and to allow for retrieval of the device in cases of prosthesis misplacement.

\section{Patient selection}

The evaluation of the potential TAVR candidate is a complex

http://www.jgc301.com; jgc@jgc301.com | Journal of Geriatric Cardiology 


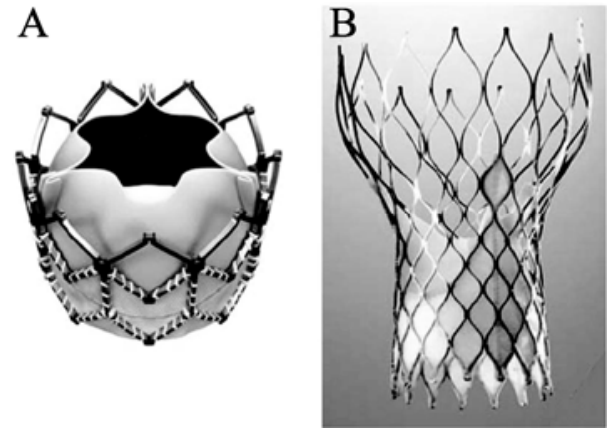

Figure 1. Currently available percutaneous aortic valves. (A): Balloon-expandable SAPIEN XT ${ }^{\circledR}$ (Edwards Lifesciences Inc., Irvine, CA, USA); (B): Self-expandable Corevalve ${ }^{\circledR}$ (Medtronic Inc., Minneapolis, MN, USA).
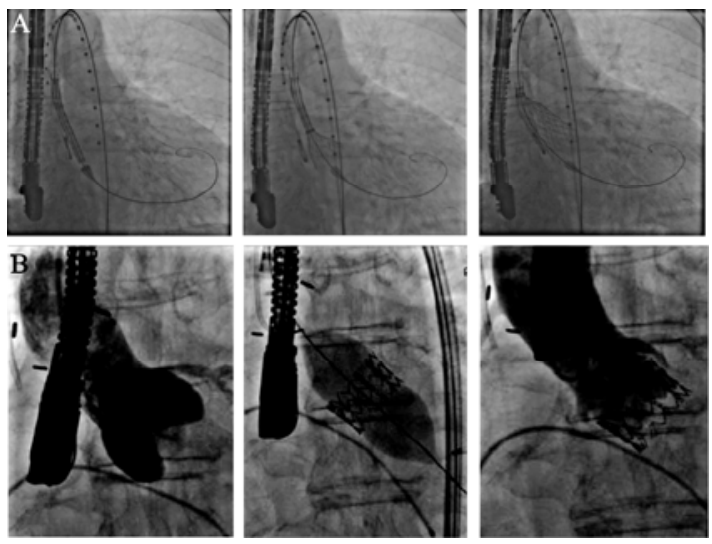

Figure 2. Valve implantation of a self-expandable (A) and a balloon-expandable (B) transcatheter valves.

process, and should be based on clinical and anatomical criteria. The diagnosis and severity of aortic stenosis should be confirmed by history, physical examination and echocardiogram (trans-thoracic and trans-esophageal). TAVR should be strictly limited to symptomatic patients, considered inoperable or at high risk for classical surgery. The surgical risk can be estimated by online risk calculators from the Society of Thoracic Surgeons (STS) (http://www.sts.org/quality-researchpatient-safety/quality/risk-calculator-and-models/risk-calculator) or the European System for Cardiac Operative Risk Evaluation (EuroSCORE, http://www.euroscore.org). The STS score tends to underestimate risk for surgical aortic valve replacement, whereas the logistic EuroSCORE overestimates risk for isolated valve surgery. Important co-morbidities known to affect surgical prognosis, such as porcelain aorta, oxygen-dependent respiratory insufficiency, cirrhosis, history of chest wall deformity or radiation, dementia and frailty, were not included in these scores, should also be considered in risk stratification.

In this regard, a multidisciplinary professional heart team approach is mandatory. Originally used in trials of selected patients with advanced coronary artery disease randomized to percutaneous coronary intervention (PCI) or coronary artery bypass grafting, ${ }^{[5]}$ the concept of a heart team approach has been extended to valvular heart disease. Ideally, the heart team should include all the professionals, such as interventional cardiologists, cardiothoracic surgeons, cardiac anesthesiologists, cardiologists and imaging specialists, involved in the procedure and care of these high risk patients with aortic stenosis. ${ }^{[6]}$

The assessment of anatomical parameters is of paramount importance for procedure success, and should involve a combination of different imaging modalities (Figure 3). The peripheral arteries (iliac/femoral) should be of sufficient size (diameter $>6 \mathrm{~mm}$ ), without excessive calcification and tortuosity. Angiography at the level of the abdominal aorta, or 3D multislice computed tomography (MSCT), is mandatory to determine the suitability of trans-femoral access. Precise measurements of the annulo-aortic complex by trans-thoracic echocardiography, trans-esophageal echocardiography or MSCT (or combination of these modalities) are required, in order to ensure correct prosthesis sizing. Coronary angiography should be performed to assess the need for PCI before or during TAVR procedure.

\section{Clinical results}

The results of several large multicenter registries ${ }^{[7-10]}$ and the prospective, randomized PARTNER trial have confirmed the safety and efficacy of this procedure and established TAVR as an alternative treatment to surgical aortic valve replacement (SAVR) in non-operable and high-risk patients.

The findings from multicenter registries in Europe and Canada $^{[7-10]}$ demonstrated that in treated patients using either SAPIEN or Corevalve systems by the trans-femoral approach, the procedural success rate was higher than $90 \%$ and mortality was below $10 \%$. Importantly, baseline factors, such as low left ventricular ejection fraction, pulmonary hypertension, severe mitral regurgitation and peri-procedural complications (cardiogenic shock, major vascular complications, stroke, and moderate-to-severe aortic regurgitation), were associated with 30-day mortality. ${ }^{[7]}$ Conversely, non-cardiac co-morbidities, such as chronic obstructive pulmonary disease, chronic kidney disease, and liver disease, are important predictors of mortality during the 1-year follow-up, with half of the deaths of noncardiac origin occurring late after TAVR.

The PARTNER trial was the first randomized study of TAVR, and included two different cohorts of patients. In the PARTNER cohort B trial, ${ }^{[11]}$ patients considered inoperable were randomized to TAVR with the Edwards valve, or to a conservative treatment (which included balloon aortic valvuloplasty). In the PARTNER cohort A trial ${ }^{[12]}$, patients 

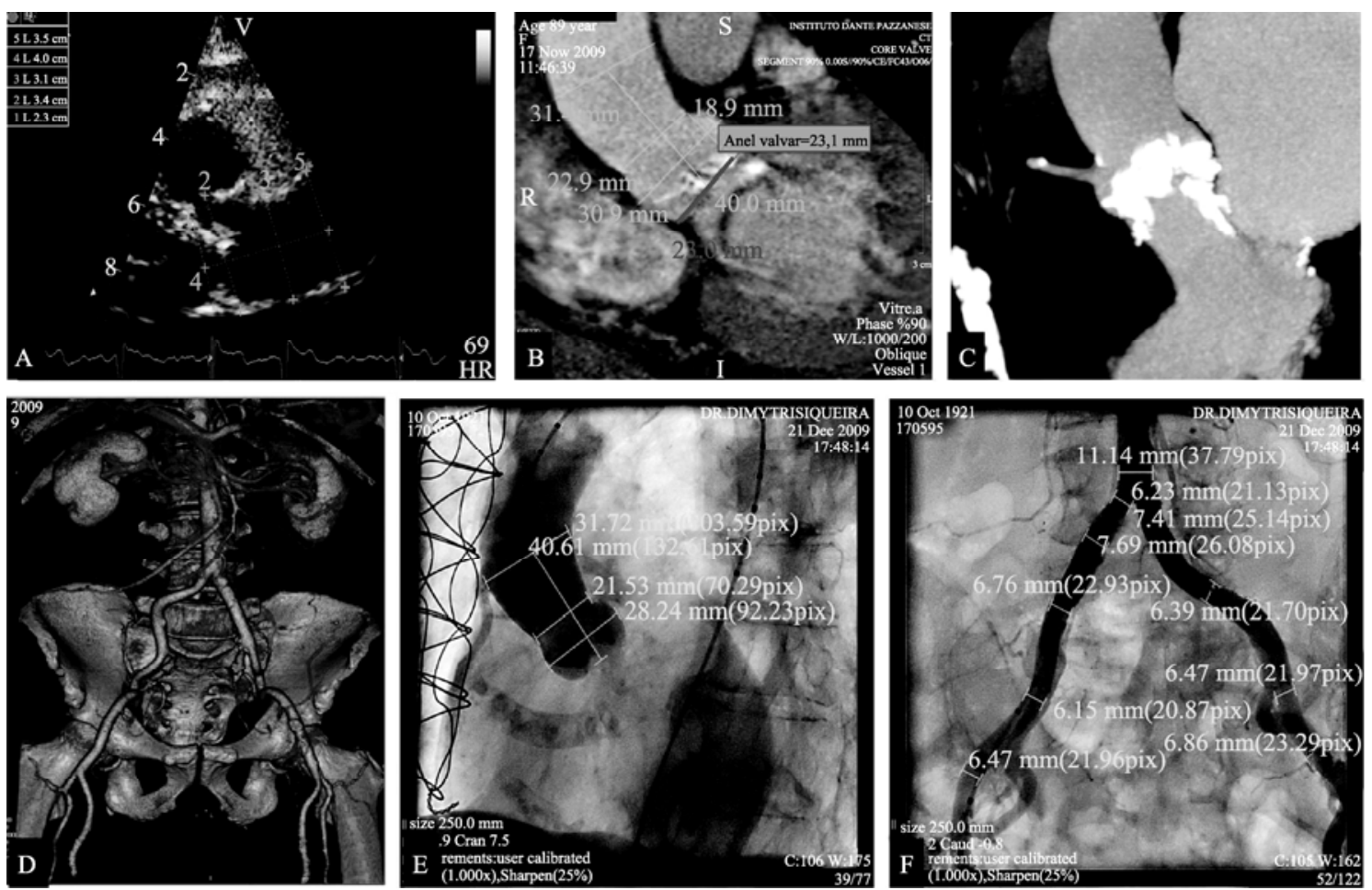

Figure 3. Multimodality imaging assessment for transcatheter aortic valve replacement. (A): Trans-thoracic echocardiography; (B\&C): Multi-slice computed tomography; (D): 3D MSTC reconstruction; (E): Aortography; and (F): Peripheral angiography.

considered to be at high surgical risk (predicted risk of operative mortality $\geq 15 \%$, or STS score $\geq 10$ ) were randomized to TAVR or SAVR. The primary end point was allcause mortality at 1-year, with the trial designed to demonstrate the superiority of TAVR vs. medical treatment for the cohort B and the non-inferiority of TAVR vs. SAVR for the cohort A.

In the non-operable cohort (PARTNER B trial), the 1-year mortality was reduced from $50.7 \%$ to $30.7 \%$ ( $P<0.0001$ ). The procedural complications in patients treated with TAVR include death (5\%), major stroke (5\%), coronary occlusion (0.6\%), vascular access site complications (16.2\%), need for permanent pacemaker (3.4\%) and moderate-to-severe aortic regurgitation (11.8\%).

In the high-risk PARTNER trial cohort A, 30-day mortality was $3.4 \%$ in the TAVR group, compared with $6.5 \%$ in the SAVR group $(P=0.07)$. Mortality at the 1 -year follow-up was $24.2 \%$ and $26.8 \%$ in the TAVR and SAVR groups, respectively ( $P=0.44$ ). At 30 days, the rates of major stroke were $3.8 \%$ in the TAVR group and $2.1 \%$ in the SAVR surgical group $(P=0.20)$ and major vascular complications occurred more significantly after TAVR (11.0\% vs. $3.2 \%, P<0.001)$.

\section{Potential complications}

\subsection{Vascular complications}

Arterial injuries (dissection, perforation, acute thrombotic occlusion) are considered the most common complications of TAVR and are often implicated, directly or indirectly, in procedural mortality. In early experiences, the relatively large diameter of the delivery catheter (22-25F) was a major limitation of trans-femoral TAVR, and higher rates of vascular complications were observed. In recent registries with the newer low-profile systems (CoreValve and Edwards NovaFlex, which are compatible with smaller 18-F sheaths), ${ }^{[7-10]}$ incidence of vascular complications varies from $2 \%$ to $17 \%$. With careful pre-procedural screening, better expertise and, in the near future, the technological improvements with smaller delivery catheter and sheath sizes (16F), further reductions in vascular injury are expected.

\subsection{Cerebrovascular accident}

The incidence of stroke varies in the published registries due to the center experience/learning curve and patient characteristics, but is also related to the definition utilized for neurological events. Accordingly, stroke rates of $0 \%-10 \%$ have been reported in association with TAVR. ${ }^{[7-10]}$ In the 
high-risk cohort of the PARTNER trial (cohort A), ${ }^{[12]}$ stroke rates tended to be higher in TAVR patients than in the SAVR group at 30 days ( $4.6 \%$ vs. $2.4 \%, P=0.12)$ and at the 1 -year follow-up (6.0\% vs. 3.2\%, $P=0.08)$.

The most frequent etiology of peri-procedural stroke is likely to be atheroembolism from the ascending aorta, or the aortic arch during balloon aortic valvuloplasty, due to valve positioning and valve deployment. However, it is believed that not all strokes are of atheroembolic origin, and postulated causes for post-procedural and late events include native valve thrombosis, atrial fibrillation, and hemorrhagic stroke.

Empiric dual oral antiplatelet therapy is currently recommended for 1 to 6 months after TAVR, followed by longterm daily low-dose aspirin. Future studies are required to evaluate safety and efficacy of dual antiplatelet therapy and the usefulness of cerebral filters devices during TAVR.

\subsection{Conduction disturbances}

Atrioventricular block, a known complication of surgical aortic valve replacement, can also occur with TAVR, presumably as a consequence of injury to the atrioventricular conduction system. As a consequence, TAVR is associated with new pacemaker implantation in $3 \%-36 \%$ of patients. ${ }^{[7-10]}$

Potential risk factors of conduction abnormalities include the presence of pre-existing infra-nodal conduction anomalies, such as right bundle branch block and deeper (too ventricular) valve implantation-particularly of the Corevalve system. Accordingly, the 9\%-36\% rate of new pacemaker implantation with the CoreValve device is higher than the 3\%-12\% rate reported with the Edwards SAPIEN device.

\subsection{Paravalvular leaks}

Hemodynamic results after TAVR, as measured by postprocedural echocardiography, are usually excellent, with the mean residual gradients $<20 \mathrm{mmHg}$ and aortic valve areas $\geq$ $1.5 \mathrm{~cm}^{2}$. These findings were superior to those obtained with stented and stentless surgical bio-prostheses, especially in female patients with a small $(<20 \mathrm{~mm})$ aortic annulus. ${ }^{[13]}$ However, although uncommon after SAVR, some degree of aortic regurgitation due to paravalvular leaks is often seen after TAVR.

Mild and even moderate degrees of paravalvular regurgitation are well tolerated. The occurrence of moderately severe or severe regurgitation, however, is often clinically significant, and has been identified as an independent predictor of mortality following TAVR. ${ }^{[14]}$ In the high-risk cohort of the PARTNER trial (cohort A), moderate or severe degree of paravalvular aortic regurgitation occurred in $12 \%$ of patients in the TAVR group, compared to $0.9 \%$ in the SAVR group $(P<0.001) .{ }^{[12]}$ A useful sign of clinical intolerance of paravalvular regurgitation is an unexpectedly low aortic diastolic pressure accompanied by a rising left ventricular filling pressure. The diagnosis is confirmed with aortography or echocardiography. The likely causes include incorrect positioning, undersizing, or underexpansion. Balloon re-expansion and valve repositioning using a snare or implantation of a second overlapping valve are recommended approaches for treatment. As mentioned, further research is needed to improve transcatheter valve technology to further reduce the incidence and severity of paravalvular leaks.

\section{Conclusions}

TAVR is a new and already mature alternative treatment for elderly patients with severe symptomatic aortic stenosis who are considered inoperable or at very high, or prohibitive, surgical risk. To date, several multicenter registries and a randomized trial have confirmed the safety and efficacy of this procedure in high risk patients. In this rapidly changing field of interventional cardiology, improvements in transcatheter valve technology, optimization of procedural results, and, hopefully, the confirmation of long-term durability of transcatheter prostheses will expand TAVR indications to a broader spectrum of patients.

\section{References}

1 Nkomo VT, Gardin JM, Skelton TN, et al. Burden of valvular heart diseases: a population-based study. Lancet 2006; 368: 1005-1011.

2 Bonow RO, Carabello BA, Chatterjee K, et al. 2008 Focused update incorporated into the ACC/AHA 2006 guidelines for the management of patients with valvular heart disease: a report of the American College of Cardiology/American Heart Association Task Force on Practice Guidelines. Circulation 2008; 118: E523-E661.

3 Iung B, Baron G, Butchart EG, et al. A prospective survey of patients with valvular heart disease in Europe: the Euro Heart Survey on valvular heart disease. Eur Heart $J$ 2003; 24: 1231-1243.

4 Cribier A, Eltchaninoff $\mathrm{H}$, Bash A, et al. Percutaneous transcatheter implantation of an aortic valve prosthesis for calcific aortic stenosis: first human case description. Circulation 2002; 106: 3006-3008.

5 Serruys PW, Morice MC, Kappetein AP, et al. Percutaneous coronary intervention versus coronary-artery bypass grafting for severe coronary artery disease. $N$ Engl J Med 2009; 360: 961-972. 
6 Holmes Jr DR, Mack MJ. Transcatheter Valve Therapy: A Professional Society Overview from the American College of Cardiology Foundation and the Society of Thoracic Surgeons. J Am Coll Cardiol 2011; 58: 445-455

7 Rodés-Cabau J, Webb JG, Cheung A, et al. Transcatheter aortic valve implantation for the treatment of severe symptomatic aortic stenosis in patients at very high or prohibitive surgical risk. Acute and late outcomes of the multicenter Canadian experience. J Am Coll Cardiol 2010; 55: 1080-1090.

8 Thomas M, Schymik G, Walther T, et al. Thirty-day results of the SAPIEN aortic bioprosthesis European outcome (SOURCE) registry: a European registry of transcatheter aortic valve implantation using the Edwards SAPIEN valve. Circulation 2010; 122: 62-69.

9 Eltchaninoff $\mathrm{H}$, Prat A, Gilard $\mathrm{M}$, et al. Transcatheter aortic valve implantation: early results of the FRANCE (FRench Aortic National CoreValve and Edwards) registry. Eur Heart J 2011; 32: 191-197.

10 Moat N, Ludman P, de Belder MA, et al. Long-term outcomes after transcatheter aortic valve implantation in high-risk patients with severe aortic stenosis. The UK TAVI (United Kingdom Transcatheter Aortic Valve Implantation) registry. J Am Coll Cardiol 2011; 58: 2130-2138.

11 Leon MB, Smith CR, Mack MJ, et al. Transcatheter aorticvalve implantation for aortic stenosis in patients who cannot undergo surgery. N Engl J Med 2010; 363: 1597-1607.

12 Smith CR, Leon MB, Mack MJ, et al. Transcatheter versus Surgical Aortic-Valve Replacement in High-Risk Patients. $N$ Engl J Med 2011; 364: 2187-2198.

13 Clavel MA, Webb JG, Rode's-Cabau J, et al. Comparison between transcatheter and surgical prosthetic valve implantation in patients with severe aortic stenosis and reduced left ventricular ejection fraction. Circulation 2010; 122: 1943-1951.

14 Tamburino C, Capodanno D, Ramondo A, et al. Incidence and Predictors of Early and Late Mortality After Transcatheter Aortic Valve Implantation in 663 Patients With Severe Aortic Stenosis. Circulation 2011; 123; 299-308. 